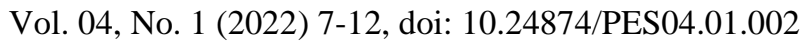 \\ Proceedings on Engineering Sciences
}

\section{VISCOUS AND MOLECULAR EFFECTS IN TRIBOCHEMICAL THIN FILM LUBRICANT FLOW}

\author{
Received 10.09.2021. \\ Accepted 02.12.2021. \\ Duriseti Venkata Srikanth ${ }^{1}$ \\ UDC - 544.722.7.023.2:532.13
}

Keywords:

Positive; Dynamic; Fatty acid; Piston; Oscilloscope.

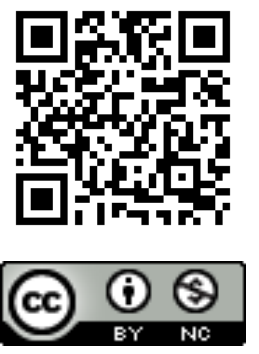

A B S T R A C T

Multiple concentrations of fatty acids in the range of $C_{10}-C_{18}$ atoms in light mineral oil were tested in this study. The experiments were carried out in a high-tech laboratory to predict pressure and flow parameters in the confines of a screw driven positive displacement piston. Theoretical and experimental data were compared for pure light mineral oil and fatty acid mixtures. The viscous forces were found to lead to the full pressure drop whereas a minor pressure drop was caused by molecular forces. With increased temperature, less pressure was required to pump the oil through the extremely thin space between parallel plates. The increase in viscosity as concentration increased, resulted in an increase of experimental strain. Variations in film temperatures showed that fluid friction was higher for the fatty acid mixture than for pure light mineral oil. For the variance of pressure with concentration, a regression analysis was plotted. The study was carried out to promote the use of surfactants in the lubricant and dynamic seal industry.

(C) 2022 Published by Faculty of Engineering

\section{INTRODUCTION}

In almost every dynamic seal design, balancing the conflicting demands of low leakage with low friction and wear of the seals' mating surfaces is a major challenge. The idea was that more tightly bound surfactant additives would provide better lubricant behaviour, resulting in a better dynamic seal. Fundamental experimental knowledge was needed in order to formulate mathematical models and predict the behaviour of thin films. Thin film migration was interpreted as flow through porous media, i.e. Darcy flow. The aim of this experiment was to compare the flow of an oil and surfactant solution to output pressure drop in the confines of a positive displacement piston and a thinly spaced (approximately $0.02 \mathrm{~mm}$ ) end-connected collection of parallel plates. The lubricant used in the study was light mineral oil, and the surfactant additives were fatty acids in the $\mathrm{C}_{10}-\mathrm{C}_{18}$ range. Common sources of hydrophobes for the non-ionic surfactant industry have been fatty acid triglycerides from both animal and vegetable sources.

In almost all cases, the main component acids belonged to the above range (Cross, 1987). The results of Palmitic acid dissolved in Polyolefin and confined between smooth surfaces were experimentally analysed (Yamada et al., 2016). The acid was primarily used as an additive friction modifier. The disparity between smooth and rough surfaces in friction behaviour was discussed. This gives insight into the formation of low-friction surfaces in the lubrication of macroscopic tribology dependent on oil. The effect of unsaturated fatty acids on the lubricating properties of a biodiesel consisting of a mixture of methyl ester fatty acids and conventional diesel fuel was studied (Martin et al., 2013).

\footnotetext{
1 Corresponding author: Duriseti Venkata Srikanth. 
The addition of fatty acids to biodiesel as additives increased the lubricating properties of the fuel at higher temperatures. Elevated temperatures and oxygen were required to shape this protective film that reduced friction and limited wear. The normal and friction forces between layers of three fatty acids and rosin acid measured in $n$ hexadecane with a surface-forced apparatus were studied (Lundgren et al., 2008). When absorbed from dry nhexadecane, almost all of the fatty acids formed loose packed monolayers on mica surfaces. In all systems, the friction force increased linearly with load, and increasing unsaturation. Friction forces were measured between molecularly smooth mica surfaces confining thin films of various branched hydrocarbons using the surface forces apparatus (Drummond \& Israelachvili, 2000). It thoroughly investigated the evolution of systems to steady-state sliding from rest or after a change in sliding velocity, noting the existence of various length and time scales. In order to fully describe the dynamic response of lubricants and complex fluids under shear, it was obvious from these findings that both time and distance of sliding had to be considered (Smith et al., 2006).

It was shown that the manufacturers of lubricants responded by producing low-viscosity lubricants with complex additive packages capable of reducing overall engine friction while preserving the engine's useful life. The friction modifier, whose task was to reduce the sliding friction of components operating in the boundary lubrication regime, was an important component in the lubricant additive package. It was widely agreed that the piston/ring/cylinder wall interface attributed to $40-60$ per cent of mechanical friction, much of which was due to the compression ring whose lubrication was kept intentionally minimal to control exhaust emissions. A computer model has been modified and used to further investigate the potential effect of the fuel administration of the friction modifier at the top ring zone. The molecular level role of the model and commercial friction modifier lubricant additives used in automatic transmissions at wet clutch interfaces were researched using surface force equipment adjusted for oscillatory shear (Zhu et al., 2003). In the boundary lubrication regime, the nano rheological properties of tetradecane were investigated with and without a model friction modifier additive and compared to a fully formulated automatic fluid transmission system (ATF). Nano rheological properties comparable to those observed for tetradecane containing $0.1-0.2$ wt. percent 1hexadecylamine were demonstrated by the ATF containing industrial grade friction modifiers. It was shown that significant improvements in the load capacity, wear resistance and friction coefficient of mechanical seals could be achieved by forming a regular microsurface structure on their surfaces in the form of micropores (Etsion,2004).

A feasibility study was performed experimentally to produce the micropores using the laser surface texture technique. Friction and face temperature had been reduced in all the tests, wear resistance had been increased and life prolonged. The study findings formed the theoretical basis for the prediction of boundary film behaviour between sliding surfaces. A model for optimising the lubricant output as well as the design of dynamic seals provided the calculated effects of various parameters. For designing more enhanced dynamic seals, the information gained from the observed effects of different lubricant constituents on the permeability factor was used. The findings of this research contributed significantly to improving the design and functioning of dynamic seals.

\section{POSITIVE DISPLACEMENT PISTON TEST RIG}

In order to analyse the flow behaviour characteristics of certain chemical fluids with and without additives such as fatty acids, the test apparatus specifically built for this project was used. Basically, this system was a long positive displacement piston operated by a digital oscilloscope and instrumentation. A cross-section of the screw-driven piston that housed the testing unit and enclosed in a chrome-steel case is shown in Fig.1. The exterior diameter and thickness of the case were $70 \mathrm{~mm}$ and $6 \mathrm{~mm}$ respectively. The lengths of the case and interior screw were $580 \mathrm{~mm}$ and $190 \mathrm{~mm}$. There was a socket at the right end of the piston into which a steel threaded plug was inserted. An annular space was formed through which a liquid was squeezed. Accurate measurements of the predetermined film thickness setting were made with two sizes of plugs.

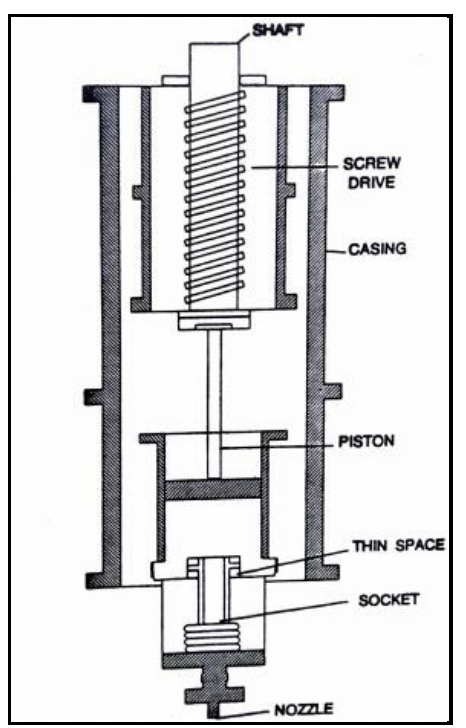

Figure 1. Cross-section of a positive displacement piston and end connected set of thin spaced parallel plates

The thin space was created by the distance between the inner surface of the socket and the outer surface of the plug (Duriseti, 1993). Between the screw drive and the piston, there was a socket inserted into which an LVDT (load cell) was inserted. After each cycle, lubricant was poured into the piston by unscrewing the right end of the 
cylinder and stripping the socket and nozzle. As it was pumped, the lubricant was drained slowly through a nozzle into the beaker.

A light sensing sensor, mounted on the shaft pulley, detected a taped spot on a white glazed background for every revolution. An end of the rpm meter was attached to the digital oscilloscope's channel B so that the signal for each cycle could be obtained. A transducer that provided an electrical output served to estimate the load or force imposed along the cell's sensitive axis. The unit configuration consisted of a compact cylinder, whose end fittings extended along the longitudinal central axis of the cylinder. A sinusoidal voltage $\mathrm{V}_{\mathrm{p}}$ with a normal frequency between $1000 \mathrm{~Hz}$ and $5000 \mathrm{~Hz}$, excited the central winding designated as the primary. There were an equivalent number of turns in the outer windings, known as secondary windings. Inside the hollow cylinder, a ferro-magnetic core rotated axially and the movement of the core shifted the magnetic flux connecting the primary winding to each of the secondary windings.

The flux variation was detected through the LVDT as in thereby establishing the basis for position measurement (Bollinger \& Duffie,1989). The secondary windings of the LVDT were in series in order to detect the voltage difference between the two windings. The magnetic flux and induced voltages in both secondary windings were equal when the core was oriented, and the voltage difference was zero. The induced voltage decreased in the other secondary winding, causing a voltage difference to appear. The output voltage was converted into readable form using an analog-digital converter. As shown in Fig.2, the different connections from the load cell to the digital oscilloscope (Nicolet Digital oscilloscope operation manual, 1982), the function generator (Hewlett Packard manual,1980) and the external jumper were used. Calibration of the load cell was done in increments of $4.536 \mathrm{~kg}$ by adding weights. This process was repeated 60 times starting from 0 to calibrate it to a maximum of $272,16 \mathrm{~kg}$ using a UTM calibrated to the US Bureau of standards.

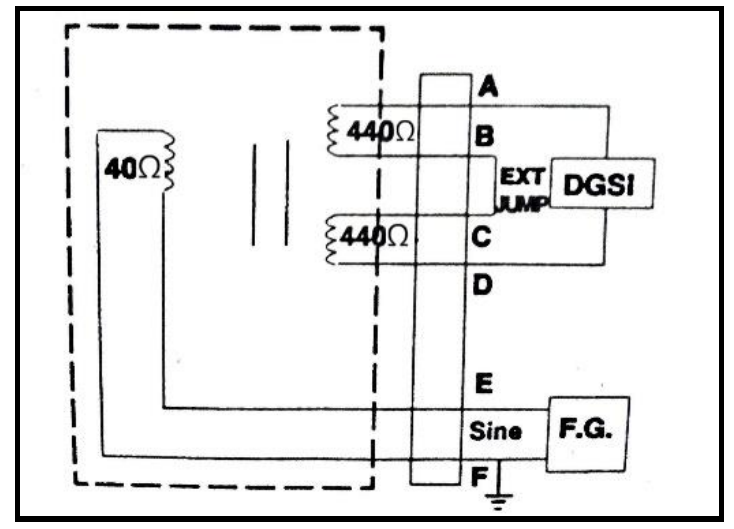

Figure 2. External circuit connecting LVDT to electronic instrumentation
A schematic representation of the various relay terminals connected to the motor, SCR DC control and 115Vac were installed as illustrated in Fig.3. ICR-1 and ICR-2 were the open contacts, ICR-3 and ICR-4 were usually closed contacts. Two momentary micro limit switches for regulating the piston motion were Limit $\mathrm{L}$ and $\mathrm{R}$.

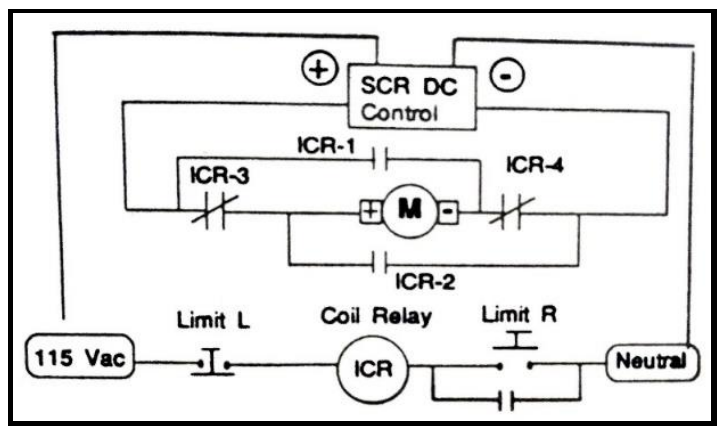

Figure 3. Schematic representation of relay connected to motor, SCR DC and $115 \mathrm{~V}$ ac.

One thing to remember was that the motor went in either direction whether the relay was driven or not. A computer control relay ladder logic circuit as in Fig. 4. was also installed.

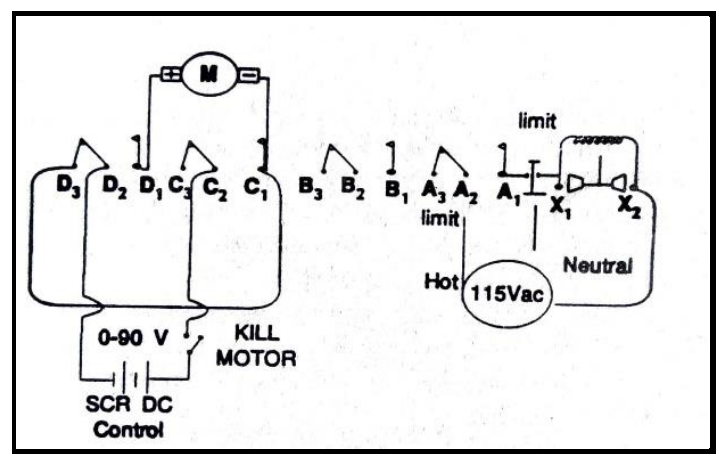

Figure 4. Machine control schematic representation (relay ladder logic circuit)

\subsection{Test Procedure:}

The step-by-step experimental procedure for each cycle corresponded to a specific flow rate, fluid, concentration and speed. Film thicknesses of $0.04 \mathrm{~mm}$ and $0.1 \mathrm{~mm}$ were obtained for the diameters of the two end-connected plugs used in the experimental sample. The displacement of the load cell read as a pressure difference was a unique feature of the test apparatus. It served as an axial load measure for each flow rate that was tested. In each cycle as lubricant was pumped, the signal as well as the AC rms voltage were registered by running programme RMS in the oscilloscope. The pitch of the screw experimentally determined was used in the actual flow rate calculation. The flow rate at any point within a boundary film was based on the pressure gradient in the theoretical predictions provided by (Fuller,1984). Local film thickness depended on pressure gradient, local velocity and a permeability factor viz. 
$P=\left(12 \mu l / b h^{3}\right) Q$

where the flow rate is $\mathrm{Q}, \mathrm{P}$ is the pressure change, $\mu$ is the viscosity, $b$ is the face width, $h$ is the thickness of the film, and 1 is the socket length. A straight-line variation in the plot was expected for a certain pressure and flow rate.

\section{RESULTS AND DISCUSSIONS}

Data obtained from the above theoretical and experimental models were compared for pure light mineral oil and fatty acid oil solutions. A composite collection of results was plotted taking all the experiments performed into account. An additional statistical regression analysis was done taking into account the variance of concentration. The measurements of the fluid film pressure for each of the $0,0.1$ and 1 percent concentrated solutions of both the well-polished plugs were done using $\mathrm{C} 10, \mathrm{C} 14, \mathrm{C} 16$ and $\mathrm{C} 18$ acids. The broad variations of the experimental and the theoretical pressures were attributed to the viscous forces and molecular forces. The findings showed satisfactory agreement between experimental and theoretical measurements for pure light mineral oil as shown in Fig.5.

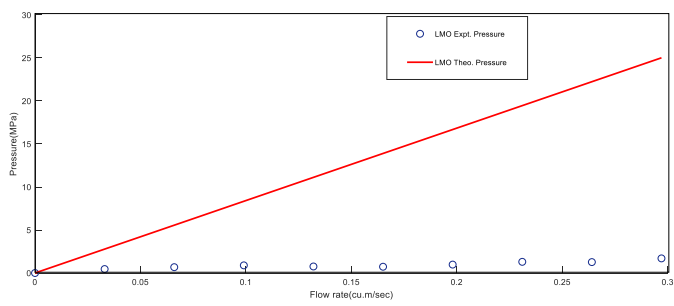

Figure 5. Comparison between the experimental and theoretical pressure profiles pure light mineral oil at $0.2 \mathrm{~mm}$ plug.

In the case of the $0.2 \mathrm{~mm}$ plug, the difference between the experimental and theoretical pressures was greater. The liquid's rise in temperature as it flowed through the annular space was high enough to see that the viscosity decreased to pump the fluid through. The data obtained for pure light mineral oil was lower than the theoretical projections for the same piston speed and film thickness, but in good agreement. Results were taken into consideration after the recalibration of the load cell was done after testing. The calibration curves prior to and after testing as shown in Fig. 6 were found to correlate well which served as validation of the results.

Fig.7 shows the theoretical pressure profiles for $0,0.1$ and $1 \%$ concentrated Palmitic acid using the $0.1 \mathrm{~mm}$ plug. It was observed that the peak pressure and the slope of the pressure profile increased with increase in concentration. This was explained by the equation

$P_{2}-P_{1}=\operatorname{loss}=f l v^{2} / 2 g D$ where

$\mathrm{f}=16 / \mathrm{Re}=16 \mu / \rho \mathrm{Vl}$

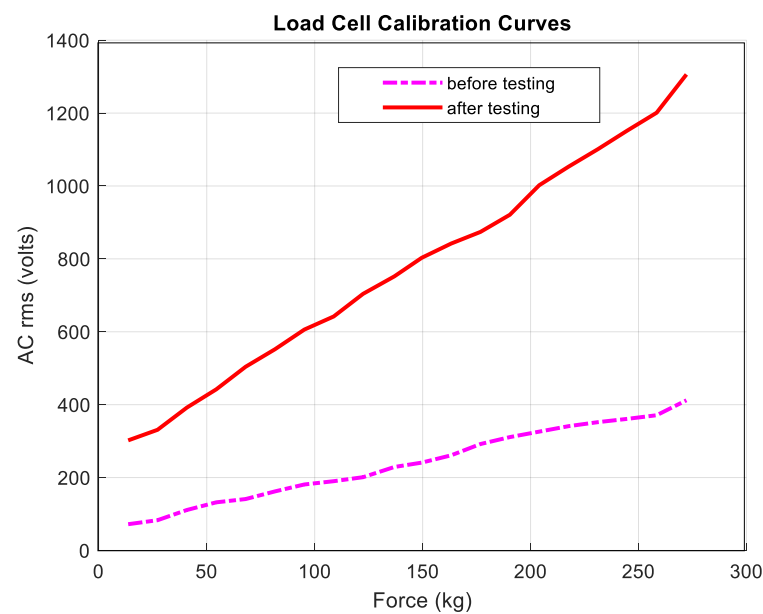

Figure 6. Validation of the calibration curves of the load cell prior to and after testing

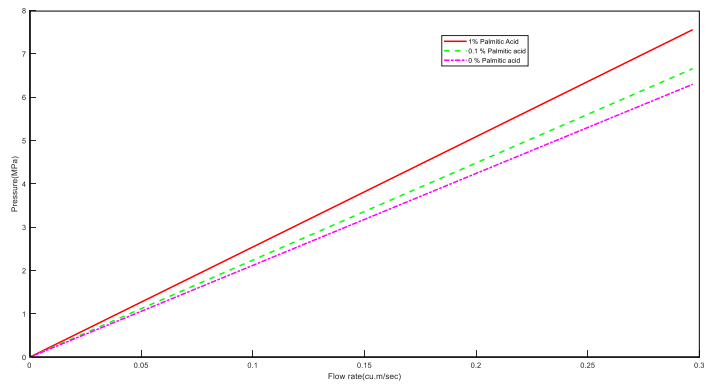

Figure 7. Comparison of the $0,0.1$ and $1 \%$ theoretical pressure profile of Palmitic acid in $0.1 \mathrm{~mm}$ plug.

With increase in concentration viz. viscosity the more friction force was generated and velocity being constant greater pressure was required to pump the fluid. Fig.8 showed the experimental and theoretical pressures for all acid mixtures of $0.1 \%$ concentration using the $0.2 \mathrm{~mm}$ plug.

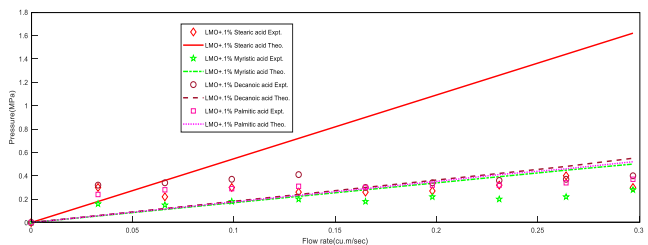

Figure 8. Combined comparison of experimental and theoretical pressures of $0.1 \% \mathrm{C}_{10}-\mathrm{C}_{18}$ acids in light mineral oil using the $0.2 \mathrm{~mm}$ plug

Similarly, Fig. 9 showed the combined experimental and theoretical pressure findings for all acid mixtures of $1 \%$ percent concentration with the $0.1 \mathrm{~mm}$ plug. The peak pressures and slopes of the pressure profiles were noted to be significant with the stearic acid blend having the highest peak pressure and pressure profile slope. 


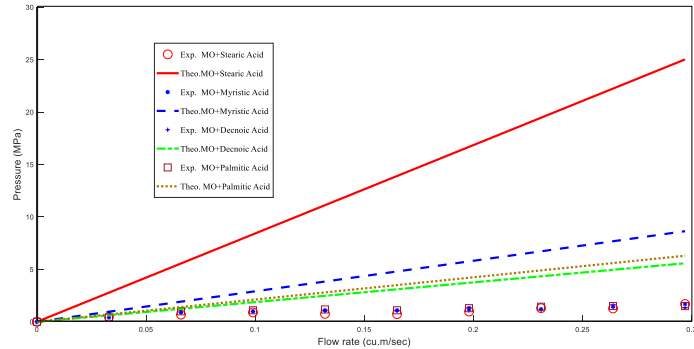

Figure 9. Combined comparison of $1 \% \mathrm{C}_{10}-\mathrm{C}_{18}$ acids with light mineral oil at $0.1 \mathrm{~mm}$ plug

Fig.10 demonstrated the concentration against pressure profiles at $0.2 \mathrm{~m}^{3} / \mathrm{sec}$ flow rate using the $0.2 \mathrm{~mm}$ size plug. The pressure vs concentration results showed that the maximum peak pressure for the said acids $\mathrm{C}_{10}-\mathrm{C}_{18}$ was justified. In the oil medium, the over emulsification of stearic acid was unique. In order to clarify the graphs, the essential micelle concentration of a surfactant decreased as the number of carbon atoms increased in the hydrophobic group to 16 (Wasan et al., 1988). Appropriate charge ions lowering the critical micelle formation was correctly attributed to the impact of lowering surface tension.

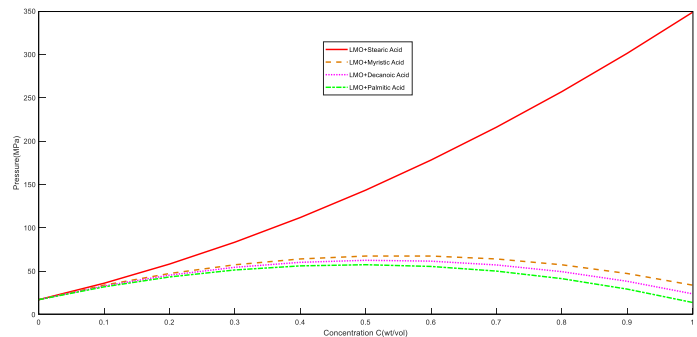

Figure 10. Pressure vs concentration curve at $0.2 \mathrm{~m}^{3} / \mathrm{sec}$ flow rate using $0.2 \mathrm{~mm}$ plug

\section{CONCLUSION}

The fluid's effective viscosity and pressure were increased with the addition of a fatty acid. The fluid friction was found to be higher with increase in fatty acid concentration. The main focus of this research was explained as the temperature of the fluid increased as it was pushed through the very thin space between parallel plates. It resulted in a decrease in viscosity-cum-pressure. In comparison to the pressure drop caused by viscous forces, the molecular forces made a minor contribution with the addition of fatty acids. Based on preliminary experimental and theoretical findings, it was determined that the given fixed geometry was useful for comparing various fluids. Under severe conditions, several phases will be encountered as the carrier lubricant begins to evaporate, complicating the linear issue. When pressure profiles of the same acid in various concentrations were grouped together, the $1 \%$ was the highest, followed by 0.1 and $0 \%$. In the presence of inorganic salts, surface and interfacial stresses decreased. The lowering of surface tension was correctly attributed to the presence of ions with the appropriate charge. These reduced the critical concentration for micelle formation. The concentration versus pressure plots indicated a justified minimum peak pressure for $\mathrm{C}_{10}-\mathrm{C}_{16}$ acids. $\mathrm{C}_{18}$ had a unique proclivity due to its excessive emulsification in the oil.

Acknowledgement: The author acknowledges the financial assistantship awarded for carrying out this study. Also grateful for the cooperation extended by the technicians in installation of equipment and arrangement of samples at the University of Florida Mechanical and Chemical Engineering Department Laboratories, Gainesville, USA.

\section{References:}

Bollinger, J. G., \& Duffie, N. A. (1989). Computer Control of Machines and Process. New York: Addison-Wesley Publishing Company.

Cross, J. (1987). Nonionic Surfactants Chemical Analysis. New York: Marcel Dekker Inc.

Drummond, C., \& Israelachvili, J. (2000). Dynamic behavior of confined branched hydrocarbon lubricant fluids under shear. Macromolecules, 33, 4910-4920.

Duriseti, S. V. (1993). Analysis of the Viscous and Molecular Effects on the flow of Tribo chemical Newtonian Fluids through a Very Thin Space between Parallel Plates (Master's Thesis, University of Florida, USA).

Etsion, I. (2004). Improving tribological performance of mechanical components by laser surface texturing. Tribology Letters, 17(4), 733-737.

Fuller, D. D. (1984). Theory and Practice of Lubrication Engineers. New York: John Wiley and Sons.

Hewlett Packard (1980). Electronic Instruments and systems, Measurement/Computation Manual. Hewlett Packard Publications, Palo Alto, pp.350-353.

Lundgren, S. M., Ruths, M., Danerlo K., \& Persson, V. K. (2008). Effects of unsaturation on film structure and friction of fatty acids in a model base oil. Journal of Colloid Interface Science. 326(2), 530-536.

Martin, J. M., Matta, C., Bouchet, M, Forest, C., Le Monge, T., Dubois, T., \& Mazarin, M. (2013). Mechanism of friction reduction of unsaturated fatty acids as additives in diesel fuels. Friction, 1(3), 252-258.

Nicolet Digital oscilloscope operation manual (1982). Nicolet Digital oscilloscope operation manual. New York, pp.1846. 
Smith, O., Priest, M., Taylor, R. I., Price, R. Cantlay, A., \& Coy, R. C. (2006) Simulated fuel dilution and friction-modifier effects on piston ring friction. Proceedings of Institution of Mechanical Engineers. Journal of Engineering Tribology. 220, 181-189.

Wasan, D. T., Ginn, M. T., \& Shah, D. . (1988). Surfactants in Chemical/Processing Engineering. New York: Marcel Dekker Inc. pp.33-125.

Yamada, S., Inomata K. A., Kobayashi, E., Tanabe, T., Kurihara K. (2016). Effect of a Fatty Acid Additive on the Kinetic Friction and Stiction of Confined Liquid Lubricants, Tribology Letters, 64(2), 1-11.

Zhu, Y., Ohtani, H., Greenfield, M. L., Ruths M., \& Granick, S., (2003). Modification of Boundary Lubrication by OilSoluble Friction Modifier Additives. Tribology Letters, 15, 127-134.

\section{Srikanth Duriseti V.}

Sreenidhi Institute of Science and

Technology,

Hyderabad

India

dvsrikanth@sreenidhi.edu.in 